Supplement of

\title{
Integrated emission inventory and modeling to assess distribution of particulate matter mass and black carbon composition in Southeast Asia
}

Didin Agustian Permadi et al.

Correspondence to: Nguyen Thi Kim Oanh (kimoanh@ait.ac.th)

The copyright of individual parts of the supplement might differ from the CC BY 3.0 License. 


\section{Supplementary Information (SI)}

Table S1. Definition of statistical measures used for WRF/CHIMERE output evaluation.

\begin{tabular}{|c|c|c|c|}
\hline Parameters & \multicolumn{2}{|l|}{ Formula } & Suggested criteria \\
\hline Mean bias $(\mathrm{MB})^{l}$ & $M B=\frac{1}{N} \sum_{i=1}^{N}\left(M_{i}-O_{i}\right)$ & & $\begin{array}{l}\mathrm{WS}: \leq \pm 0.5 \mathrm{~m} \mathrm{~s}^{-1} \\
\mathrm{WD}: \leq \pm 10 \mathrm{deg} \\
\mathrm{T} \quad: \leq \pm 0.5^{\circ} \mathrm{C} \\
\mathrm{H} \quad: \leq \pm 1 \mathrm{~g} \mathrm{~kg}^{-1}\end{array}$ \\
\hline $\begin{array}{l}\text { Mean absolute gross error } \\
(\mathrm{MAGE})^{I}\end{array}$ & $M A G E=\frac{1}{N} \sum_{i=1}^{N}\left|M_{i}-O_{i}\right|$ & & $\begin{array}{l}\text { WD }: \leq+30 \mathrm{deg} \\
\mathrm{T} \quad: \leq+2{ }^{\circ} \mathrm{C} \\
\mathrm{H} \quad: \leq+2 \mathrm{~g} \mathrm{~kg}^{-1}\end{array}$ \\
\hline $\begin{array}{l}\text { Root mean squared error } \\
(\mathrm{RMSE})^{l}\end{array}$ & $R M S E=\sqrt{\frac{1}{N} \sum_{i=1}^{N}\left(M_{i}-O_{i}\right)^{2}}$ & & $\mathrm{WS}: \leq+2 \mathrm{~m} \mathrm{~s}^{-1}$ \\
\hline Mean fractional bias(MFB $)^{2}$ & $M F B=\frac{2}{N} \sum_{1}^{N}\left[\frac{\left(M_{i}-O_{i}\right)}{\left(M_{i}+O_{i}\right)}\right]$ & .100 & PM criteria : $\leq \pm 60 \%$ \\
\hline Mean fractional error (MFE) ${ }^{2}$ & $M F E=\frac{2}{N} \sum_{1}^{N}\left[\frac{\left|M_{i}-O_{i}\right|}{\left(M_{i}+O_{i}\right)}\right.$ & .100 & PM criteria $: \leq+75 \%$ \\
\hline
\end{tabular}

Notes: Adapted from the compilation by Kim Oanh et al. (2012)

$\mathrm{M}$-modeled value (model outputs for the first layer in different grids); O-observations; N-number of observations; WS-Wind Speed; WD-Wind Direction; T-Temperature; H- Relative Humidity.

Sources: ${ }^{1}$ Emery et al. (2001), ${ }^{2}$ Boylan and Russell (2006). 
Table S2. Summary of ground observations used for model evaluation.

\begin{tabular}{|c|c|c|c|c|c|}
\hline \multirow[t]{2}{*}{ No } & \multirow[t]{2}{*}{ Data sources } & \multirow[t]{2}{*}{ Data type } & \multicolumn{3}{|c|}{ Information } \\
\hline & & & Name & longitude & latitude \\
\hline \multicolumn{6}{|c|}{ Meteorological parameters } \\
\hline 1 & Olongapo-Phillippines & TMP, RH, DIR, SPD & RPLB-98426 & 14.8 & 120.3 \\
\hline 2 & Davao-Phillippines & TMP, RH, DIR, SPD & RPMD-98753 & 7.1 & 125.6 \\
\hline 3 & Don Muang-Thailand & TMP, RH, DIR, SPD & VTBD-48456 & 13.9 & 100.6 \\
\hline 4 & Trat-Thailand & TMP, RH, DIR, SPD & VTBO-48500 & 12.3 & 102.3 \\
\hline 5 & Pnom Penh-Cambodia & TMP, RH, DIR, SPD & VDPP-48991 & 11.5 & 104.8 \\
\hline 6 & Jakarta-Indonesia & TMP, RH, DIR, SPD & WIII-96749 & 6.1 & 106.7 \\
\hline 7 & Kuala Lumpur-Malaysia & TMP, RH, DIR, SPD & WMKK-48647 & 3.1 & 101.5 \\
\hline 8 & Sarawak-Malaysia & TMP, RH, DIR, SPD & WBKK-96471 & 5.9 & 116.0 \\
\hline \multicolumn{6}{|c|}{ Ground measurement of aerosols } \\
\hline 1 & AIRPET project & $\begin{array}{l}\text { Daily }(24 \mathrm{~h}) \text { average } \\
\text { of } \mathrm{PM}_{10}, \mathrm{PM}_{2.5}, \mathrm{BC} \text {, } \\
\text { and } \mathrm{OC} \text { ambient } \\
\text { concentrations }\end{array}$ & $\begin{array}{l}\text { - AIT, Thailand (sub- } \\
\text { urban) } \\
\text { - Tegallega (TL), } \\
\text { Indonesia (mix } \\
\text { urban) } \\
\text { - Manila (MO), } \\
\text { Philippines } \\
\text { observatory (mix } \\
\text { urban) } \\
\text { - Thuong Dinh (TD), } \\
\text { Vietnam (mix } \\
\text { urban) }\end{array}$ & $\begin{array}{l}100.6 \\
107.6 \\
121.1 \\
105.8\end{array}$ & $\begin{array}{l}14.1 \\
-6.9 \\
14.6\end{array}$ \\
\hline 2 & APN project & $\begin{array}{l}\text { Hourly EC/BC and } \\
\text { OC }\end{array}$ & $\begin{array}{l}\text { AIT, Thailand (sub- } \\
\text { urban) }\end{array}$ & 100.6 & 14.1 \\
\hline 4 & $\begin{array}{l}\text { Urban air quality } \\
\text { monitoring network in } \\
\text { Bangkok, Thailand }\end{array}$ & Hourly $\mathrm{PM}_{10}$ & $\begin{array}{l}\text { Klongjun (10T), } \\
\text { Thailand (mix urban) } \\
\text { Huaykwang (11T), } \\
\text { Thailand (mix urban) }\end{array}$ & $\begin{array}{l}100.5 \\
100.6\end{array}$ & $\begin{array}{l}13.8 \\
13.9\end{array}$ \\
\hline 5 & $\begin{array}{l}\text { Urban air quality } \\
\text { monitoring network in } \\
\text { Kuala Lumpur, Malaysia }\end{array}$ & Hourly $\mathrm{PM}_{10}$ & $\begin{array}{l}\text { - Petaling Jaya, } \\
\text { Malaysia (Urban) } \\
\text { - Jerantut, } \\
\text { Malaysia(remote) } \\
\end{array}$ & $\begin{array}{l}101.6 \\
101.7\end{array}$ & $\begin{array}{l}3.1 \\
3.1\end{array}$ \\
\hline 6 & $\begin{array}{l}\text { City air quality } \\
\text { monitoring network in } \\
\text { Surabaya, Indonesia }\end{array}$ & Hourly $\mathrm{PM}_{10}$ & $\begin{array}{l}\text { Taman Prestasi (SUF1) } \\
\text { Indonesia (mix urban) }\end{array}$ & 112.7 & -7.3 \\
\hline \multicolumn{6}{|c|}{ Ground measurement of AOD } \\
\hline 1 & AERONET Thailand & AOD level 2 & Phimai & 102.6 & 15.2 \\
\hline 2 & AERONET Singapore & AOD level 2 & Singapore & 103.8 & 1.3 \\
\hline 3 & AERONET Vietnam & AOD level 2 & Bac Giang & 106.2 & 21.3 \\
\hline 4 & AERONET Vietnam & AOD level 2 & Bac Lieu & 105.7 & 9.3 \\
\hline 5 & AERONET Taiwan & AOD level 2 & Chen Kung & 120.2 & 23.0 \\
\hline 6 & AERONET Hong Kong & AOD level 2 & Hok Sui & 114.3 & 22.2 \\
\hline 7 & AERONET Thailand & AOD level 2 & Mukdahan & 104.7 & 16.6 \\
\hline 8 & AERONET Indonesia & AOD level 2 & Puspitek Serpong & 106.6 & 6.3 \\
\hline 9 & AERONET Thailand & AOD level 2 & Silpakorn University & 100.0 & 13.8 \\
\hline 10 & AERONET Thailand & AOD level 2 & Songkhla Met Station & 100.6 & 7.2 \\
\hline
\end{tabular}


Table S3. Summary of simulated domain maximum ground-level concentrations $\mathrm{PM}_{10}, \mathrm{PM}_{2.5}$ and $\mathrm{BC}$ for different periods.

\begin{tabular}{|c|c|c|c|c|c|c|c|c|c|c|c|c|c|}
\hline \multirow[b]{2}{*}{ Pollutants } & \multirow[b]{2}{*}{ Averaging period $^{\mathrm{a}}$} & \multicolumn{12}{|c|}{ Month, 2007} \\
\hline & & Jan & Feb & Mar & Apr & May & Jun & Jul & Aug & Sept & Oct & Nov & Dec \\
\hline \multirow{3}{*}{$\begin{array}{c}\mathrm{PM}_{10} \\
\left(\mu \mathrm{g} \mathrm{m}^{-3}\right)\end{array}$} & Max hourly & 325 & 267 & 157 & 327 & 242 & 253 & 261 & 245 & 240 & 259 & 164 & 162 \\
\hline & Max monthly avg & 69 & 89 & 65 & 127 & 62 & 48 & 72 & 58 & 69 & 86 & 44 & 30 \\
\hline & Max annual avg & \multicolumn{12}{|c|}{50} \\
\hline \multirow{3}{*}{$\begin{array}{c}\mathrm{PM}_{2.5} \\
\left(\mu \mathrm{g} \mathrm{m}^{-3}\right)\end{array}$} & Max hourly & 188 & 154 & 157 & 189 & 143 & 147 & 147 & 150 & 139 & 150 & 99 & 98 \\
\hline & Max annual avg & 40 & 55 & 49 & 82 & 47 & 27 & 44 & 37 & 45 & 53 & 27 & 19 \\
\hline & Annual average & \multicolumn{12}{|c|}{32} \\
\hline \multirow{3}{*}{$\begin{array}{c}\mathrm{BC} \\
\left(\mu \mathrm{g} \mathrm{m}^{-3}\right)\end{array}$} & Max hourly & 39 & 31 & 28 & 38 & 30 & 31 & 33 & 32 & 30 & 32 & 23 & 20 \\
\hline & Max monthly avg & 8.2 & 10.1 & 21.1 & 15.8 & 7.1 & 6.1 & 9.3 & 7.8 & 8.7 & 10.9 & 5.9 & 2.1 \\
\hline & Max annual avg & \multicolumn{12}{|c|}{6} \\
\hline
\end{tabular}

Note: ${ }^{\text {a }}$ One maximum value simulated in the whole modeling domain for the considered period. 


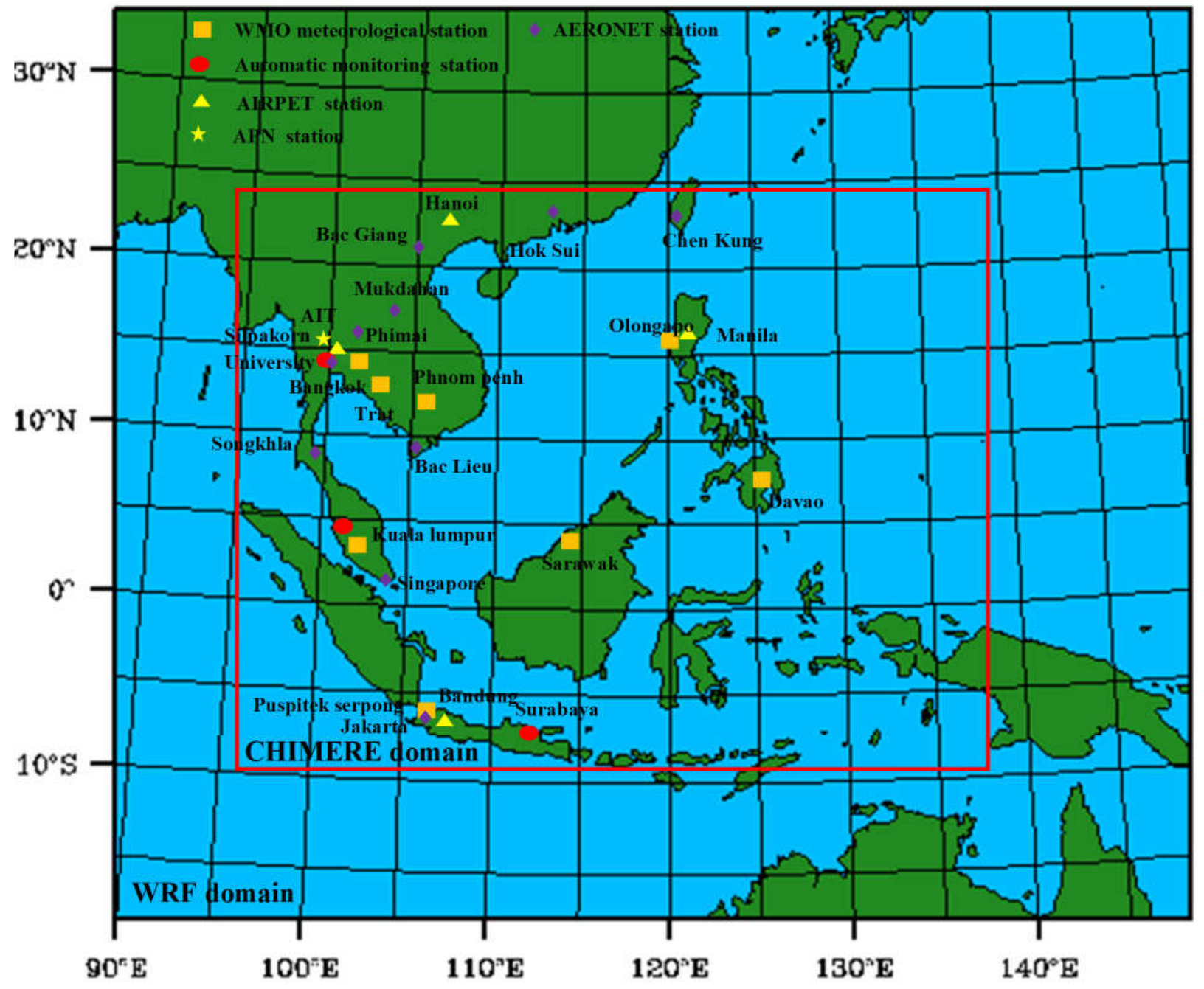

Figure S1. Modeling domains of WRF and CHIMERE. 

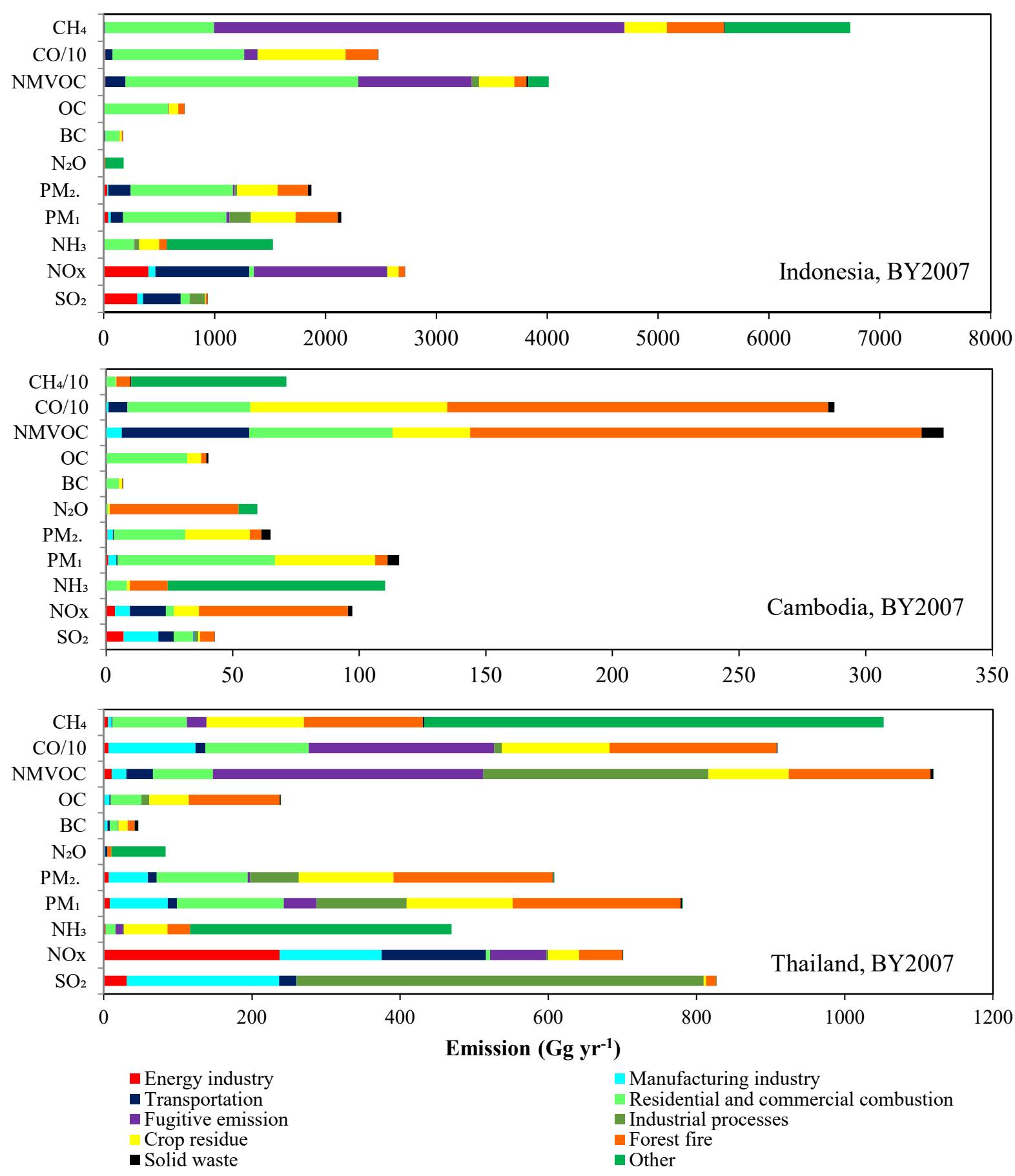

Figure S2. Total emission and shares for Indonesia, Cambodia and Thailand for base year of 2007. 

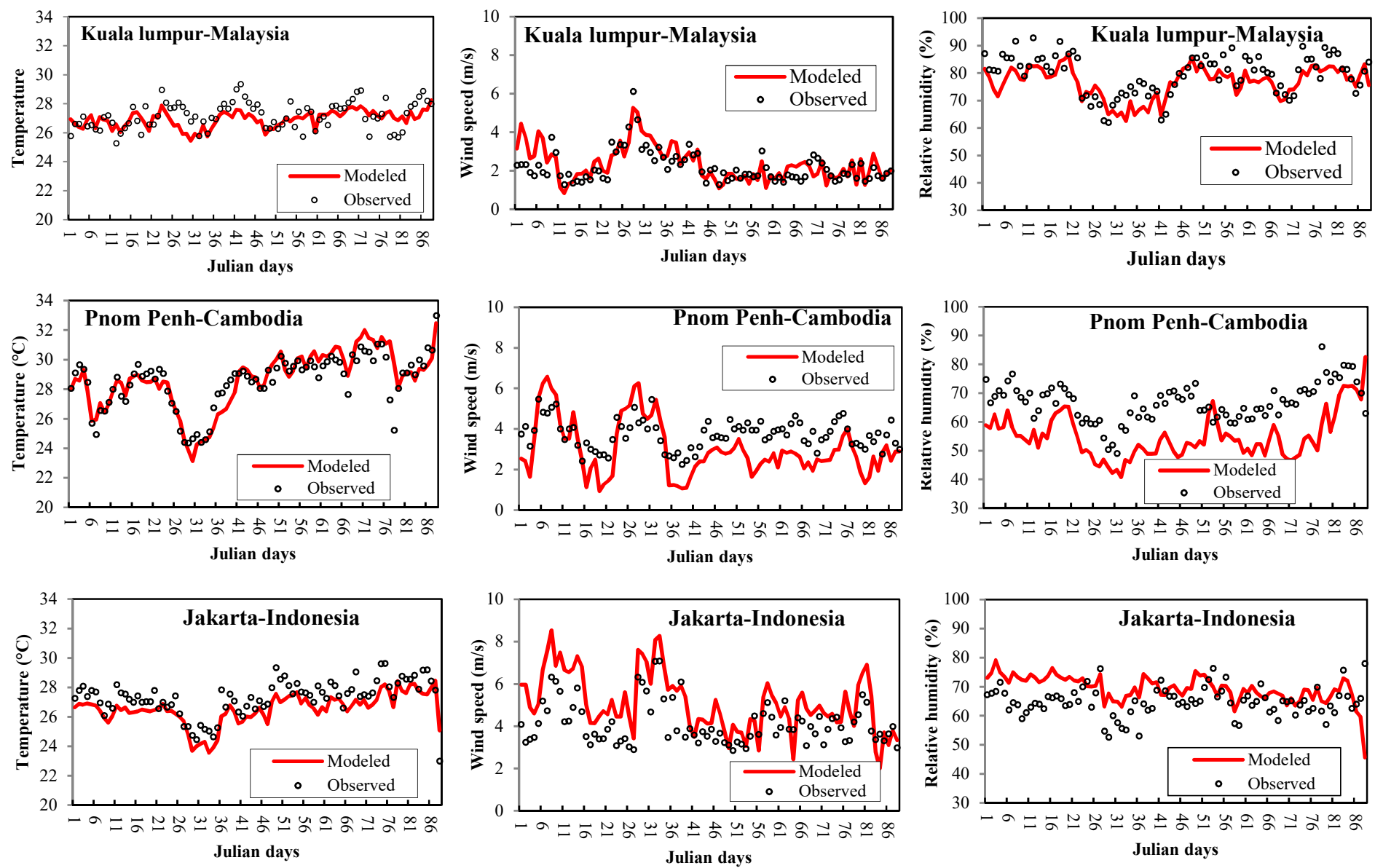

Figure S3a. Time series of daily average modeled (WRF) vs. observed relative humidity, temperature and wind speed at three WMO stations in Kuala Lumpur (Malaysia), Phnom Penh (Cambodia), and Jakarta (Indonesia) for period of January - March 2007. 

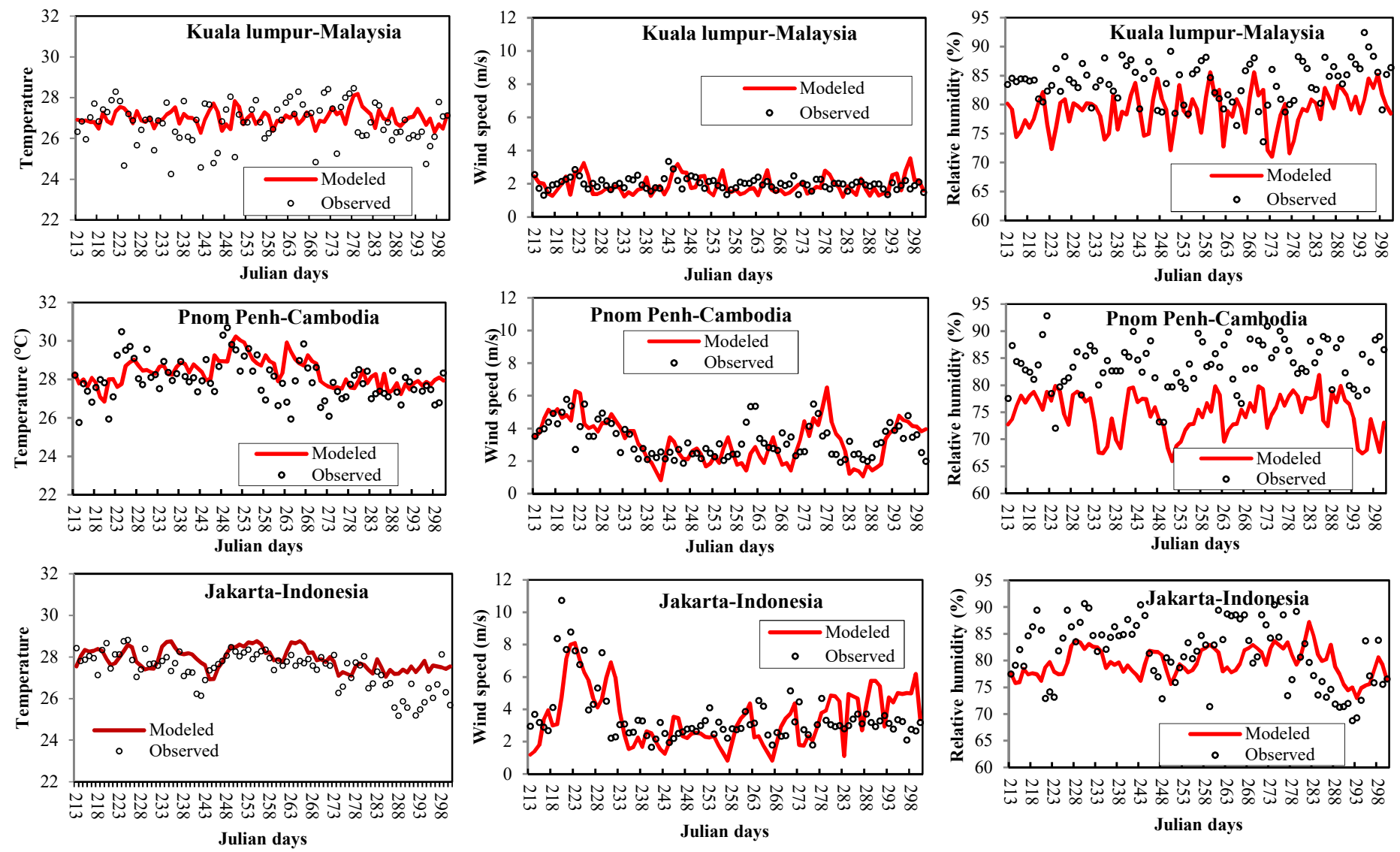

Figure S3b. Time series of daily average modeled (WRF) vs. observed relative humidity, temperature and wind speed at three WMO stations in Kuala Lumpur (Malaysia), Phnom Penh (Cambodia), and Jakarta (Indonesia) for period of August - October 2007. 

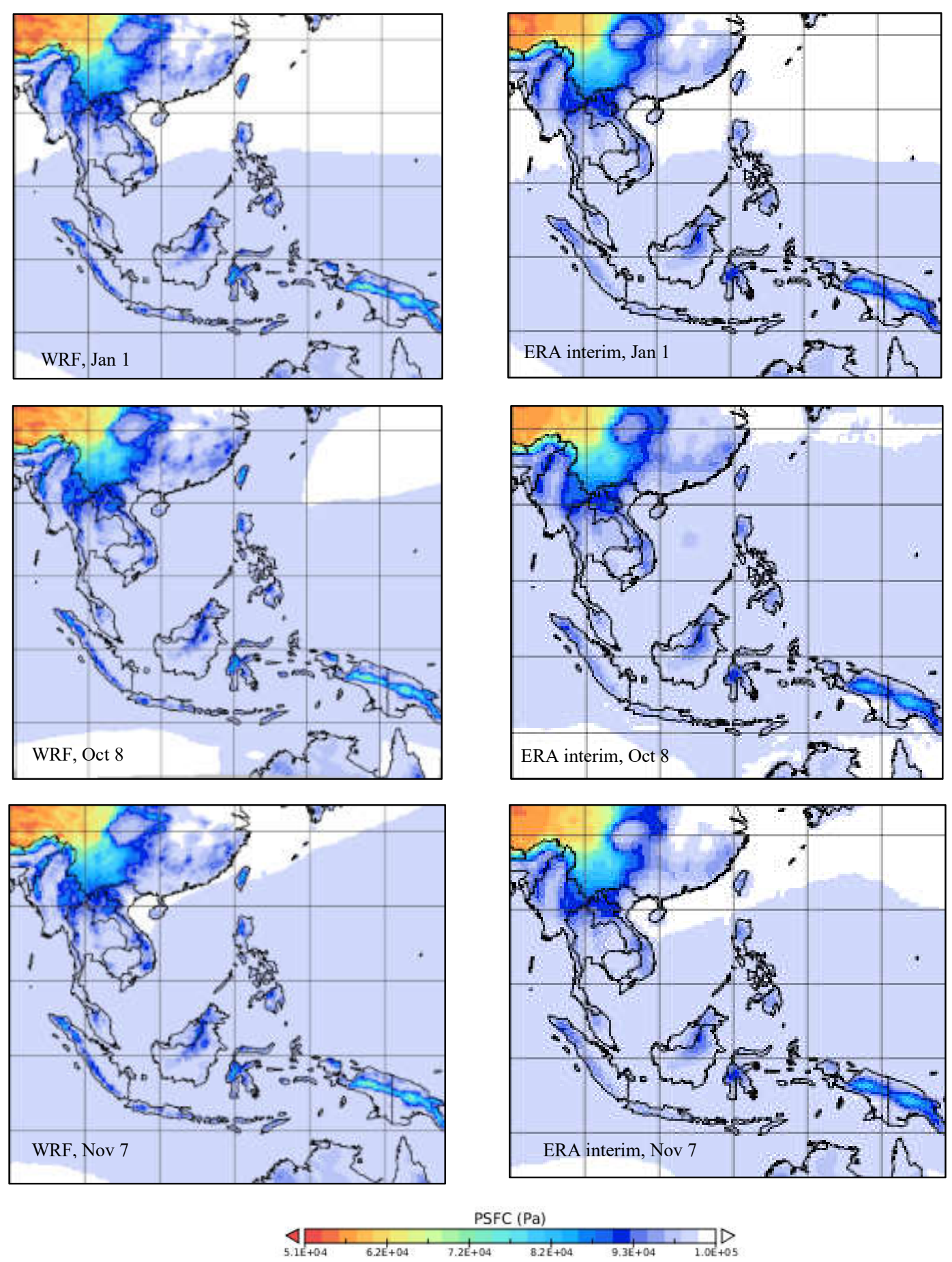

Figure S4. Comparison of modeled surface pressure and ERA interim reanalysis data for the selected days in 2007 at 00:00 UTC. 

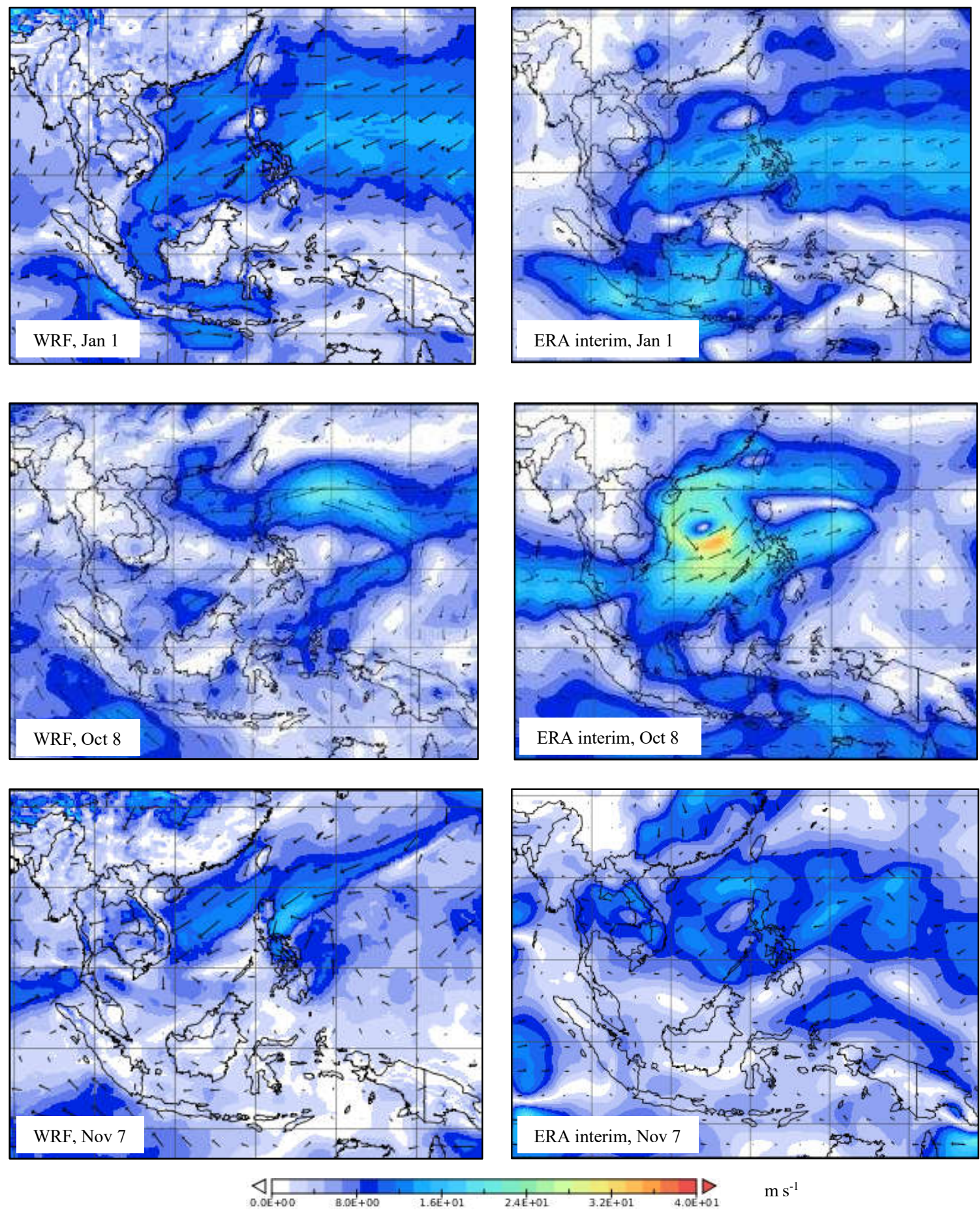

Figure S5. Comparison of modeled and ERA upper wind fields at $850 \mathrm{hPa}$ on the selected days in 2007 at 00:00 UTC. 


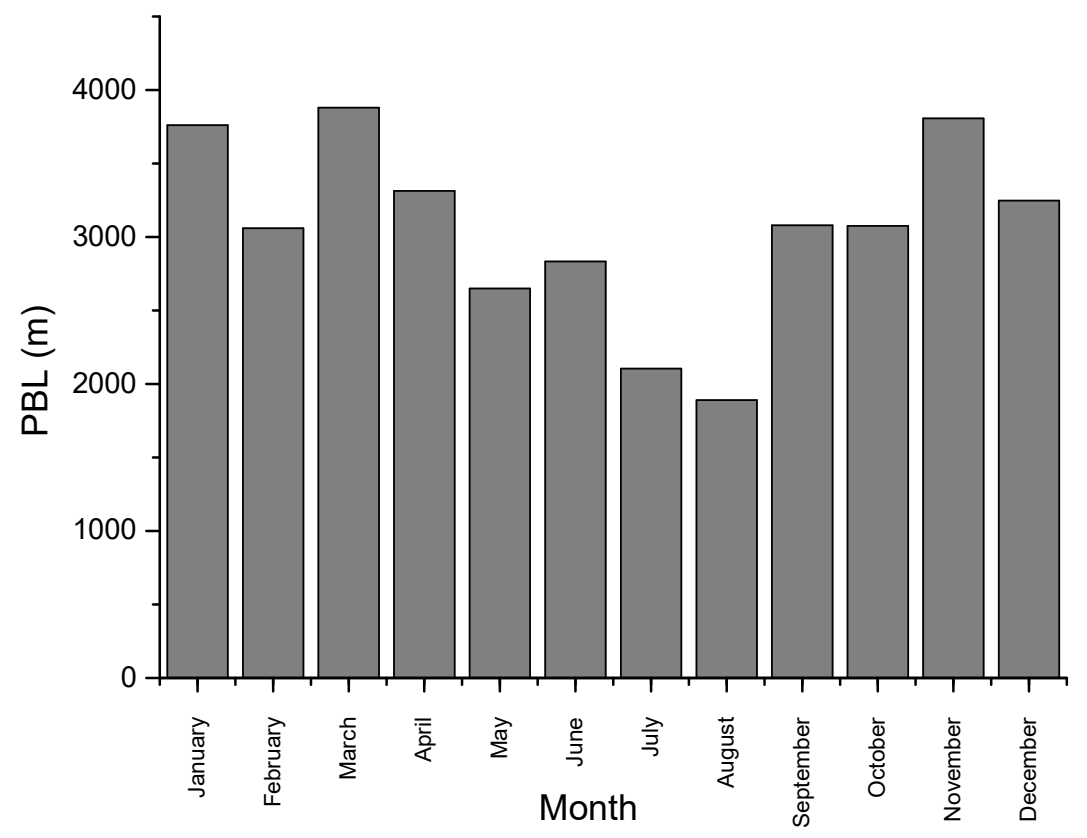

Figure S6. Domain maximum hourly values of simulated PBL for the different months. 

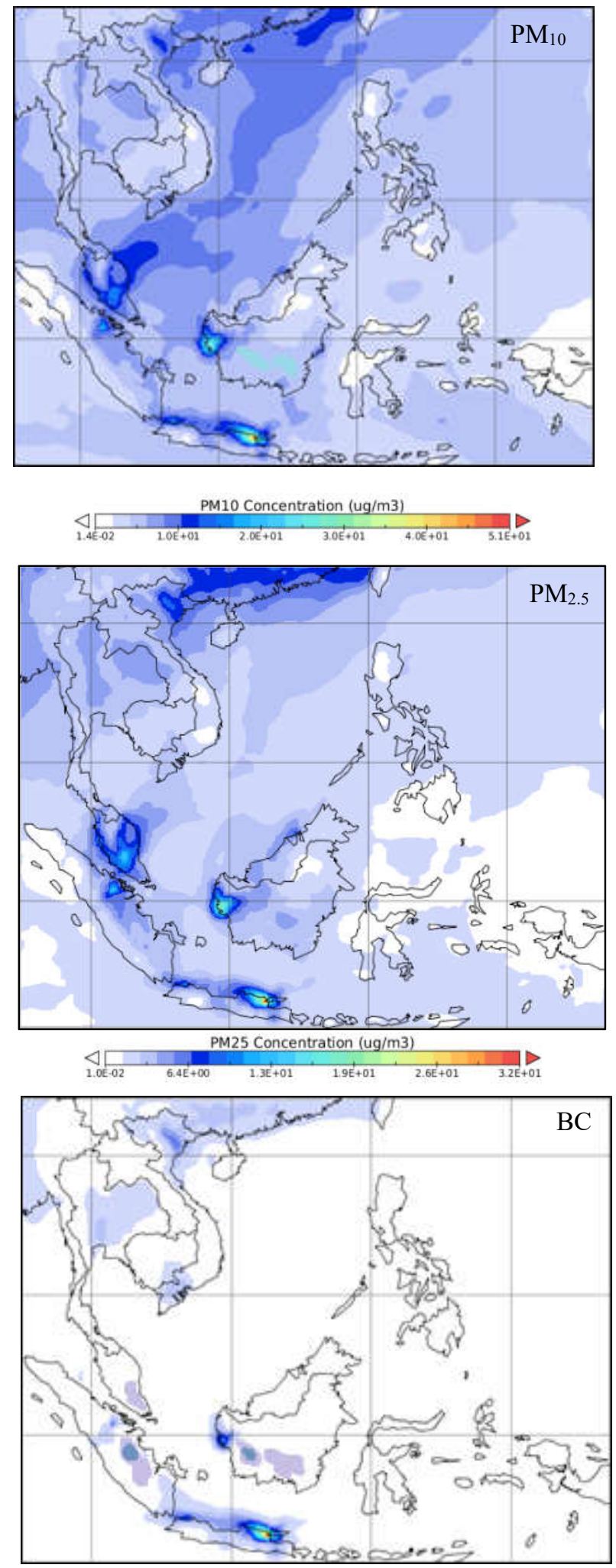

$B C$ Concentration (ug/m3)

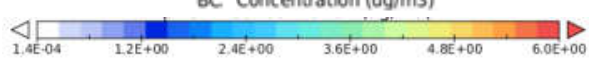

Figure S7. Simulated annual average of PM10, $\mathrm{PM}_{2.5}$ and $\mathrm{BC}$ concentrations of 2007 over the domain. 

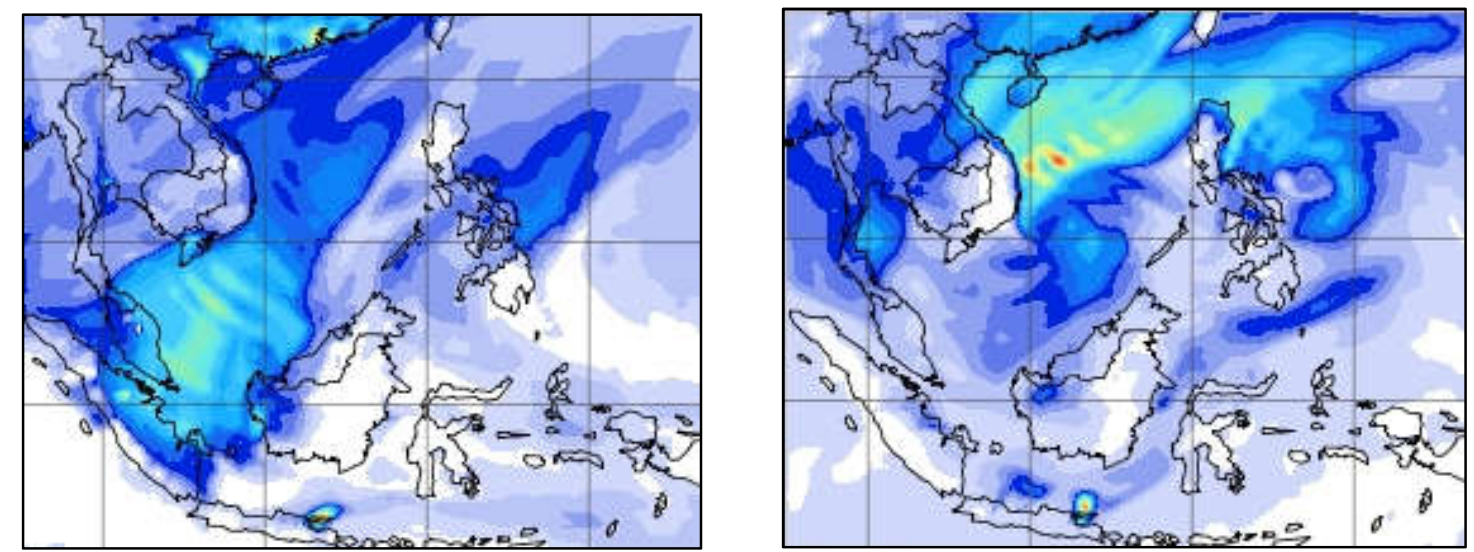

a) $\mathrm{PM}_{10}, 22 \mathrm{Jan}$

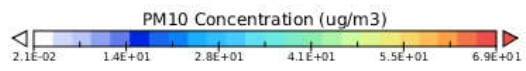

b) $\mathrm{PM}_{10}, 20 \mathrm{Nov}$
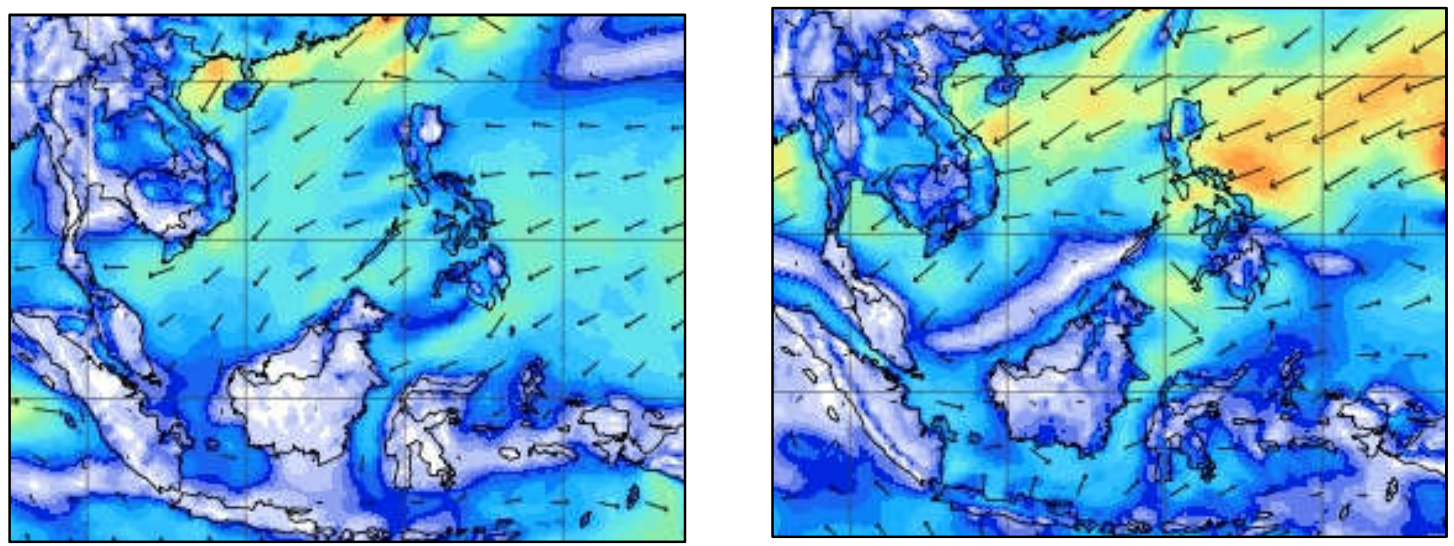

c) Wind field (10 m), 22 Jan

$\underset{6.6 E-03}{4}{ }_{3.0 E+00}^{\operatorname{Sqrt}\left[(\mathrm{U} 10)^{2}+(\mathrm{V} 10)^{2}\right](\mathrm{m} \mathrm{s}-1)}$

d) Wind field (10 m), 20 Nov
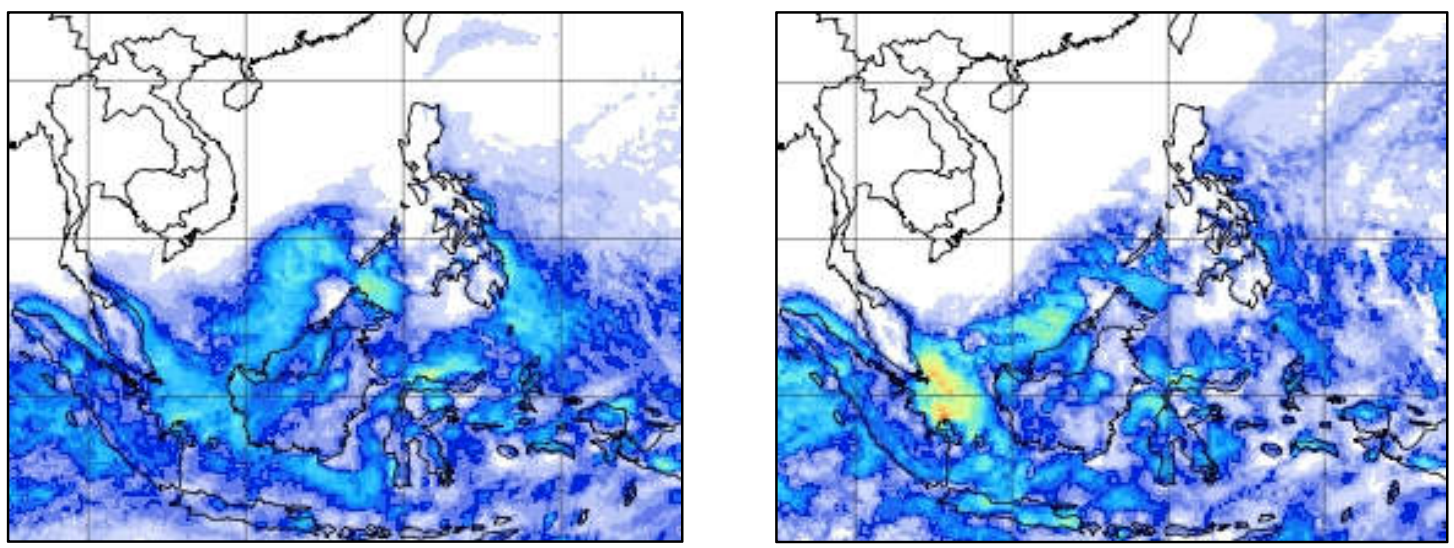

e) Hourly precipitation, 22 Jan

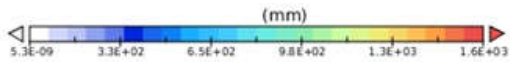

f) Hourly precipitation, $20 \mathrm{Nov}$

Figure S8. Typical simulated maximum hourly $\mathrm{PM}_{10}$ concentration and wind field at $10 \mathrm{~m}$ in 22 January and 20 November, 00:00 UTC. 

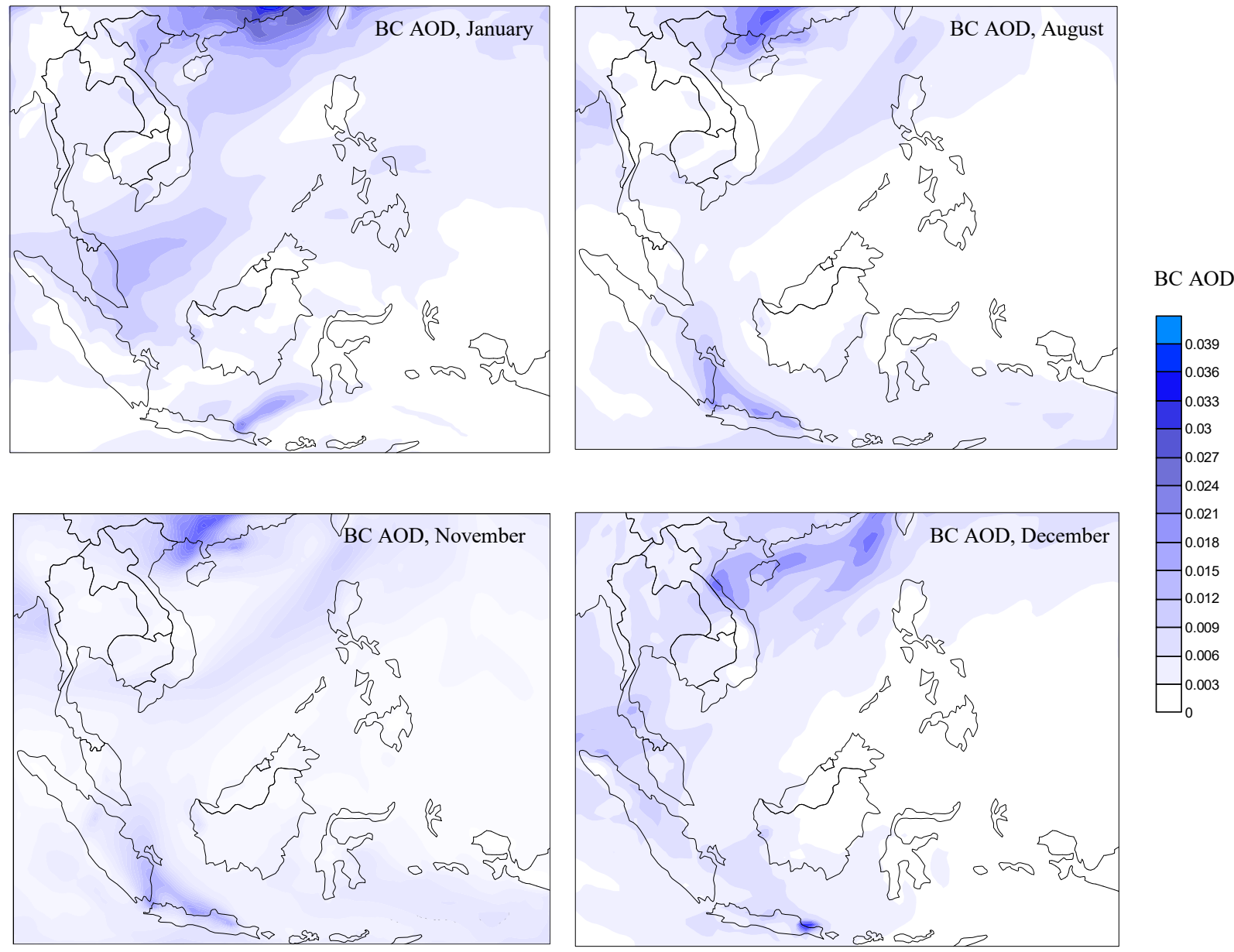

Figure S9. Monthly average of simulated BC AOD for the selected months. 